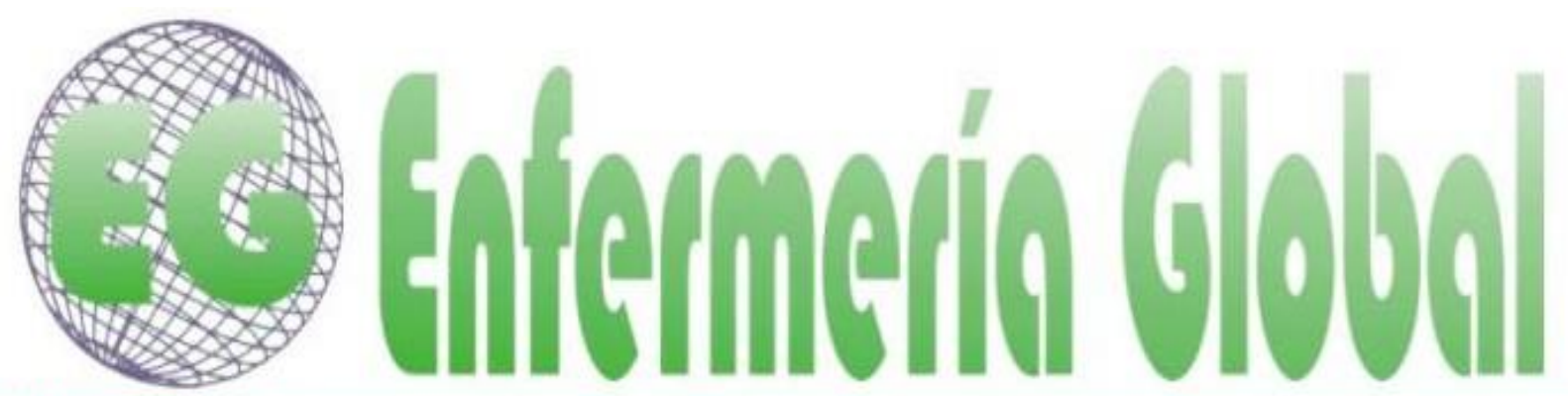

\title{
Índice de vulnerabilidad al VIH en población habitante de calle
}

HIV vulnerability index in homeless persons

\section{*Berbesi Fernández, Dedsy **Segura Cardona, Ángela *** Cardona Arango, Doris ${ }^{* * * *}$ Caicedo Velasquez, Beatriz}

*Enfermera. PhD Epidemiología y Bioestadística. Docente Facultad de Medicina. E-mail:
dberbesi@ces.edu.co ${ }^{* *}$ Estadística. PhD Epidemiología. Docente Facultad de Medicina.
${ }^{* * *}$ Administradora. PhD Demografía. Docente Facultad de Medicina. ****PhD Geografía Humana. Docente Facultad de Medicina. Universidad CES. Medellín-Colombia.

http://dx.doi.org/10.6018/eglobal.16.2.252001

\section{RESUMEN}

Objetivo. Se reconoce que las vulnerabilidades sociales tienden a ocurrir con el $\mathrm{VIH}$, pero pocos estudios han tratado de medir la vulnerabilidad al VIH y no se logra identificar en la literatura la unificación frente al concepto y la manera de operativizarlo. El objetivo de esta investigación fue determinar un índice de vulnerabilidad, y su relación con la infección por VIH en población habitante de calle de la ciudad de Medellín

Material y método. Se realizó un estudio descriptivo transversal con 338 habitantes de calle de Medellín utilizando el muestreo de tiempo y lugar. En la construcción del índice se realizó un análisis factorial de tipo exploratorio y un análisis factorial confirmatorio.

Resultados. Se identificaron cuatro factores que explicaron el 50,49\% de la varianza. Se encontró que la población con alta vulnerabilidad al VIH tenía cinco veces el riesgo de tener $\mathrm{VIH}$, esta asociación fue ajustada por variables sociodemográficas de edad, sexo, estado civil y nivel de escolaridad.

Conclusiones. Se propone un índice de vulnerabilidad al VIH, el cual se definió como la reducción de la capacidad de anticiparse (conocimientos y creencias erróneas), resistirse (prácticas sexuales y consumo de drogas) y recuperarse (apoyo social y rechazo). Lo cual limita la capacidad para acceder a servicios de prevención, atención, y apoyo al VIH.

Palabras clave: VIH; Poblaciones Vulnerables; Condiciones Sociales; Personas sin hogar (Términos DeCs) 


\section{ABSTRACT}

Purpose: While there is evidence that social vulnerabilities tend to co-occur with HIV, few studies have measured HIV vulnerability. To date, there is no consensus in the literature regarding how to measure or operationalize HIV vulnerability. Therefore, the purpose of this study was to determine an HIV vulnerability index in homeless persons in the city of Medellín, Colombia.

Material and methods: This cross-sectional study included 338 homeless persons in the city of Medellín, Colombia, and time-location sampling was used. During the construction of the index, an exploratory factor analysis, and a confirmatory factor analysis (CFA) were performed.

Results: Four factors accounting for $50,49 \%$ of the variance were identified. The population with high HIV vulnerability had a five-fold greater risk of being infected with HIV. This association was adjusted for socio-demographic variables including age, gender, civil status, and education level.

Conclusions. We propose an HIV vulnerability index that is defined as the reduced ability to anticipate (knowledge and erroneous beliefs), resist (sexual practices and drug use), and recover (social support and rejection), which limits the ability to access HIV prevention, attention, and support services.

Keywords: HIV; Vulnerable Populations; Social Conditions; Homeless Persons

\section{INTRODUCCIÓN}

El Virus de Inmunodeficiencia Humana VIH sigue siendo un importante problema de salud pública mundial, después de haber cobrado más de 36 millones de vidas y estimarse para el 201235 millones de personas infectadas por el VIH (1). En Colombia, la población más afectada por la epidemia es la población joven en el grupo de los 15 a 34 años de edad con el 56,5\% de los casos notificados por VIH/Sida y muerte, seguido de los mayores de 45 años con el 15,2\% de la participación ${ }^{(2)}$.

Aunque en los inicios de la epidemia existió una rápida propagación del VIH/Sida, surgió independiente de las condiciones socioeconómicas y demográficas, pues avanzó simultáneamente en los países desarrollados y en los países en desarrollo, pero la trayectoria para su irrupción perdió ese carácter transversal, ya que las comunidades con mayor grado de desarrollo lograron contenerla, pero las más pobres y con menor nivel de educación continúan sufriendo sus efectos más severos ${ }^{(3)}$.

Los primeros análisis de la situación del VIH partieron del enfoque biológico, centrando su mirada en el comportamiento de cada individuo, y determinándolos como grupos de riesgo ${ }^{(4,5)}$. De acuerdo con estos criterios, se hicieron encuestas que indagaban sobre conductas individuales que sirvieron para construir lo que se categorizó como factores de riesgo ${ }^{(6,7)}$.

Queriendo superar el enfoque individual, y reconociendo que para entender el comportamiento de la epidemia eran fundamental el contexto de vida de las personas $(8,9)$, se reconsideraron los factores de riesgo configurándolos como problemas sociales y se les dio el nombre de comportamientos de riesgo; pero debido a la ausencia de un entendimiento amplio de la población en riesgo para VIH en sus contextos sociales y culturales, los alcances de este enfoque fueron limitados ${ }^{(3)}$.

Se plantearon entonces modelos con los elementos frecuentemente excluidos, y en los últimos años, partiendo de la comprensión del VIH más allá de las condiciones individuales y atendiendo a los contextos sociales y de exclusión descritos, se ha empezado a realizar análisis en poblaciones específicas cuyas características 
sociales, culturales y de condición individual pueden aumentar el riesgo de la infección ${ }^{(10)}$.

Desde ese momento, se empezaron a definir los grupos vulnerables al VIH/Sida como aquellos individuos que practicaban conductas de riesgo o que no se protegían adecuadamente $^{(11)}$; pero a medida que la enfermedad se fue propagando, los grupos vulnerables fueron definidos por la capacidad de protegerse frente a conductas riesgosas, y esa capacidad está estrechamente relacionada con las condiciones sociales, culturales y demográficas ${ }^{(12)}$. Es decir, se reconoció que la infección por VIH es una fuente de vulnerabilidad social, con mayores consecuencias en los grupos con menores ventajas ${ }^{(13)}$.

A pesar de las indicaciones de que las vulnerabilidades sociales tienden a co-ocurrir, pocos estudios han tratado de medir la vulnerabilidad al VIH. No se logra identificar en la literatura la unificación frente al concepto y por lo tanto la manera de operativizarlo (14). Este manuscrito propone un índice de vulnerabilidad al VIH en población habitante de calle; tomando como referente lo descrito por Ayres en sus componentes individuales y sociales; dentro de la dimensión individual se identifican: creencias, conocimientos, actitudes y en la dimensión social las redes sociales, el uso de drogas, el rechazo, la discriminación y el soporte social ${ }^{(15)}$.

También se incluyen categorías del modelo AVEO (acrónimo para Activos, Vulnerabilidad social y Estructura de Oportunidades) el cual plantea, ampliar la mirada sobre el problema de pobreza ${ }^{(16)}$, De esta manera, la vulnerabilidad se define como la interrelación que se da entre dos niveles: la estructura de oportunidades (conocimientos previos, consumo de drogas y apoyo social) y los activos (habilidades para negociar y no tener conductas sexuales de riesgo). Por lo anterior, se buscó construir un índice de vulnerabilidad al VIH para la población habitante de calle, que aporte a la identificación, prevención y control de la epidemia. Este artículo hace parte de un trabajo de tesis doctoral donde se elaboró este concepto y se identificó la prevalencia de VIH en la población habitante de calle ${ }^{(17)}$.

\section{MATERIAL Y MÉTODO}

Se realizó un estudio descriptivo transversal, utilizando fuente de datos primarios derivados de la aplicación de un instrumento a 338 habitantes de calle de la ciudad de Medellín en el primer semestre de 2014.

Inicialmente se realizó la construcción de reactivos para el índice, se identificaron los constructos objeto de estudio (el contenido), tomando como referencia artículos que exploraron la medición de la vulnerabilidad al VIH ${ }^{(15,18-20)}$. La revisión bibliográfica y el análisis de estudios previos, conformaron la plataforma sobre la que se construyó el cuestionario de vulnerabilidad al $\mathrm{VIH}$.

La técnica fue la encuesta dirigida y el instrumento consistió en un formulario con preguntas demográficas y sociales del habitante de calle y una prueba para la detección de $\mathrm{VIH}$; previo consentimiento informado; la entrevista la realizaba una persona experta en consejería para VIH y enfermedades de transmisión sexual.

El cuestionario fue aplicado a los habitantes de calle, utilizando el muestreo de tiempo y lugar conocido por sus siglas en inglés como TLS (Time Location Sampling). Este método es utilizado en poblaciones de difícil acceso. Para el cálculo del tamaño de la 
muestra se estimó una población de 3.381 habitantes de calle de acuerdo al censo realizado en Medellín en el año $2009^{(21)}$, utilizando una proporción de habitantes de calle con VIH de 7,8\% ${ }^{(22),}$ un efecto de diseño del 1,5\% y una precisión del $4 \%$. Los requerimientos éticos fueron aprobados por el Comité Institucional de Ética de la Universidad CES de Medellín-Colombia en el acta nro. 55 de 2013.

En la construcción del índice se realizó un análisis factorial de tipo exploratorio, mediante el método de componentes principales con rotación Varimax. Previamente se transformaron las variables cualitativas a través del método de cuantificación óptima y se calculó el coeficiente alfa de Cronbach para cada uno de los factores encontrados.

Puntaje de Vulnerabilidad al VIH. Para facilitar la interpretación del constructo de vulnerabilidad al VIH, se siguió lo descrito por Grisales ${ }^{(23,24)}$, se tipificó de modo que el rango posible de valores estuviera entre $X$ mínimo $=0$ y $X$ máximo=100 puntos; de esta forma, a mayor puntaje, mayor vulnerabilidad al VIH. La tipificación se hizo conociendo el menor y mayor valor ( $Z$ min y $Z$ máx.) de las componentes.

Se llevó a cabo también un Análisis Factorial Confirmatorio, en el cual se analizaron las cargas factoriales del constructo, las estimaciones estandarizadas y los estadísticos de bondad de ajuste, se utilizó el estimador de máxima verosimilitud. La finalidad principal del análisis factorial no fue el estudio de relaciones causales, sino la agrupación de categorías en función de la variabilidad que cada variable compartía con las demás ${ }^{(25)}$.

Finalmente se realizó un modelo multivariado de regresión logística binaria utilizando como variable dependiente tener o no la infección por $\mathrm{VIH}$ con el fin de identificar la fuerza de asociación con el índice de vulnerabilidad al VIH, esta relación fue ajustada por variables sociodemográficas. Estos análisis se realizaron en el programa SPSS 21.0 licencia de la Universidad CES.

\section{RESULTADOS}

\section{Características demográficas}

Fueron encuestados 338 habitantes de calle con edades entre 18 y 65 años, con edad promedio de 40,0 $\pm 11,4$ años. El $50 \%$ de ellos contaba con 41 años o menos y el $75 \%$ con 50 años o menos. El mayor nivel educativo encontrado fue primaria, lo que significa que la población en su mayoría solo tenía una educación básica. Predominó el estado civil soltero, con el 71,3\% (241), seguido con una menor proporción por los separados o divorciados (16,3\%), en unión libre (8,6\%) y casados $(2,7 \%)$. Relacionado con el sexo, las mujeres tienen una proporción siete veces mayor en la condición civil de unión libre, mientras los hombres superan a las mujeres en la condición de soltería y de separados o divorciados. La prevalencia de VIH fue de 8,15\% (IC 95\% 3,92- 12,37).

\section{Construcción de reactivos y cuestionario}

En la construcción del cuestionario fueron analizados 45 reactivos: 1) Una escala de conocimientos de $\mathrm{VIH}$, de 19 ítems, utilizada en dos estudios previos, con una consistencia interna buena (alfa de Cronbach $=0,94$ ) y adecuadas propiedades psicométricas medidas a través de un análisis factorial exploratorio ${ }^{(26,27)}$. 2) Variables 
sobre comportamientos de riesgo sexual, aplicadas previamente en población habitante de calle ${ }^{(22)}$. 3) Escala sobre la frecuencia de consumo de drogas, aplicada en sistemas de vigilancia del consumo de drogas (VESPA). 4) Redes sociales, en este factor se analizan las redes que apoyan a la población habitante de calle y el rechazo y discriminación que reciben (tabla 1 ).

Tabla 1. Análisis factorial exploratorio. Construcción del índice de vulnerabilidad al VIH

\begin{tabular}{|c|c|c|c|c|}
\hline Variables & $\begin{array}{c}\text { Conocimientos, } \\
\text { creencias y } \\
\text { actitudes }\end{array}$ & $\begin{array}{l}\text { Consumo } \\
\text { de drogas }\end{array}$ & $\begin{array}{c}\text { Redes } \\
\text { sociales y } \\
\text { rechazo }\end{array}$ & $\begin{array}{l}\text { Comportamiento } \\
\text { de riesgo sexual }\end{array}$ \\
\hline \multicolumn{5}{|c|}{ El VH se transmite (Conocimientos incorrectos) } \\
\hline Al tener relaciones sexuales sin usar condón & 0,92 & & & \\
\hline Al recibir una transfusión sanguínea & 0,88 & & & \\
\hline Por usar agujas de otra persona & 0,88 & & & \\
\hline Teniendo una sola pareja & 0,83 & & & \\
\hline Al evitar utilizar jeringas usadas & 0,82 & & & \\
\hline Absteniéndose de tener relaciones sexusles & 0,81 & & & \\
\hline Utilizando el condón siempre & 0,77 & & & \\
\hline De la mujer embarazada infectada al bebe & 0,73 & & & \\
\hline Teniendo solo sexo oral & 0,60 & & & \\
\hline Al retirar el condón & 0,56 & & & \\
\hline De madre al hijo por la lactancia matems & 0,56 & & & \\
\hline Existe la reinfección por $\mathrm{VlH}$ & 0,55 & & & \\
\hline \multicolumn{5}{|l|}{ EI VH se transmite (Creencias erróneas) } \\
\hline Al dar la mano o abrazar & 0,85 & & & \\
\hline Al usar beños públicos & 0,74 & & & \\
\hline Al compartir cubiertos & 0,73 & & & \\
\hline Al besar una persona con VIH & 0,71 & & & \\
\hline Al compartir una comida & 0,67 & & & \\
\hline Por la picadura de un mosquito & 0,51 & & & \\
\hline \multicolumn{5}{|c|}{ Cree que una persona con ИH (actitudes ante el ИH) } \\
\hline Puede tener VH y lucir bien & 0,73 & & & \\
\hline \multicolumn{5}{|l|}{ Frecuencia del consumo de drogas } \\
\hline Consumo de hongos & & 0,77 & & \\
\hline Consumo de Popper & & 0,75 & & \\
\hline Consumo de cacao sabanero & & 0,73 & & \\
\hline Consumo de ruedas & & 0,71 & & \\
\hline Consumo de cocsína & & 0,54 & & \\
\hline Consumo de inhalantes & & 0,47 & & \\
\hline Consumo de marihuana & & 0,39 & & \\
\hline \multicolumn{5}{|l|}{ Rechazo de la población } \\
\hline Ha sido maltratado en los Servicios de salud & & & 0.46 & \\
\hline Ha recibido desprecio, rechazo u ofensas & & & 0,44 & \\
\hline Ha sufrido algún abuso o maltrato por ser hab. & & & 0,42 & \\
\hline \multicolumn{5}{|l|}{ Apoyo recibido } \\
\hline Tiene el apoyo de alguien & & & 0,80 & \\
\hline Tiene apoyo en caso de ser maltratado & & & 0,83 & \\
\hline Tiene apoyo en caso de tener VH & & & 0,83 & \\
\hline \multicolumn{5}{|l|}{ Uso del Condón } \\
\hline Uso del condón en relaciones orales & & & & 0,68 \\
\hline Uso del condón en relaciones penetrativas & & & & 0,64 \\
\hline Uso del condón en relaciones vaginales & & & & 0,80 \\
\hline
\end{tabular}

\section{Descripción del índice}

Se identificaron cuatro factores: el primer factor denominado conocimientos, creencias, $y$ actitudes ante el VIH explicó el 29,9\% de la varianza, el segundo factor explicó el $8,6 \%$ y se denominó consumo de drogas; el tercer factor se categorizó como rechazo, discriminación y falta de redes sociales y explicó el 6,6\% de varianza, y el cuarto factor se denominó consumo de comportamientos de riesgo, el cual explicó 
el 5,3\% de la varianza (Tabla 1). El test KMO (Kaiser, Meyer y Olkin), el cual mide la relación entre las variables analizadas, donde valores cercanos a 1 implica una alta relación entre las variables, mostró un valor de 0,91 , indicando que existe una relación adecuada entre las variables del estudio. La prueba de esfericidad de Bartlett del modelo, la cual evalúa la aplicabilidad del análisis factorial de las variables estudiadas, fue significativo $(p<0,00)$. Los cuatro factores explicaron el $50,49 \%$ de la varianza del constructo vulnerabilidad al $\mathrm{VIH}$.

\section{Consistencia interna}

El índice de vulnerabilidad al VIH presentó una consistencia interna adecuada con un alfa de Cronbach de 0,86; por sub-escalas el alfa de Cronbach también fue adecuado: 0,95 en la sub-escala de conocimientos, creencias y actitudes al VIH; en la sub-escala de consumo de drogas el alfa de Cronbach fue de 0,75; y las sub-escalas de comportamientos de riesgo y redes sociales obtuvieron un alfa de Cronbach de 0,6 cada una.

\section{Concepto de Vulnerabilidad al VIH}

Con el índice de vulnerabilidad construido, y la revisión bibliográfica realizada, se diseñó una nueva propuesta teórica, utilizando como referente lo descrito en vulnerabilidad y medio ambiente, que considera que la vulnerabilidad, se produce cuando las relaciones entre seres vivos y el medio físico, rompen la estabilidad que garantiza la capacidad de anticiparse, resistirse y adaptarse o evolucionar con los fenómenos naturales ${ }^{(28)}$.

Se utilizaron las tres categorías descritas en esta definición, y se adaptaron a las variables observadas e incluidas en el índice de vulnerabilidad; las variables conocimientos al VIH y creencias erróneas, la cual había sido descrita como prevención del VIH tomó la denominación de anticiparse a la infección, los ítems de consumo de drogas y no uso del condón, se agruparon y la variable latente se describió como resistirse; y por último el no tener apoyo social y ser rechazado y discriminado, conformaron la categoría de la capacidad de adaptarse a la infección por VIH.

Este nuevo modelo teórico, representado en un esquema explicativo, se lleva a un análisis factorial confirmatorio (AFC), el cual buscaba posibilitar una mejor comprensión de la realidad y de los fenómenos observados, y determinar mediante pruebas estadísticas, en qué medida los datos de la muestra apoyaban el modelo propuesto de vulnerabilidad al VIH (Figura 1). 
Figura 1. Modelo AFC del Concepto de Vulnerabilidad al VIH

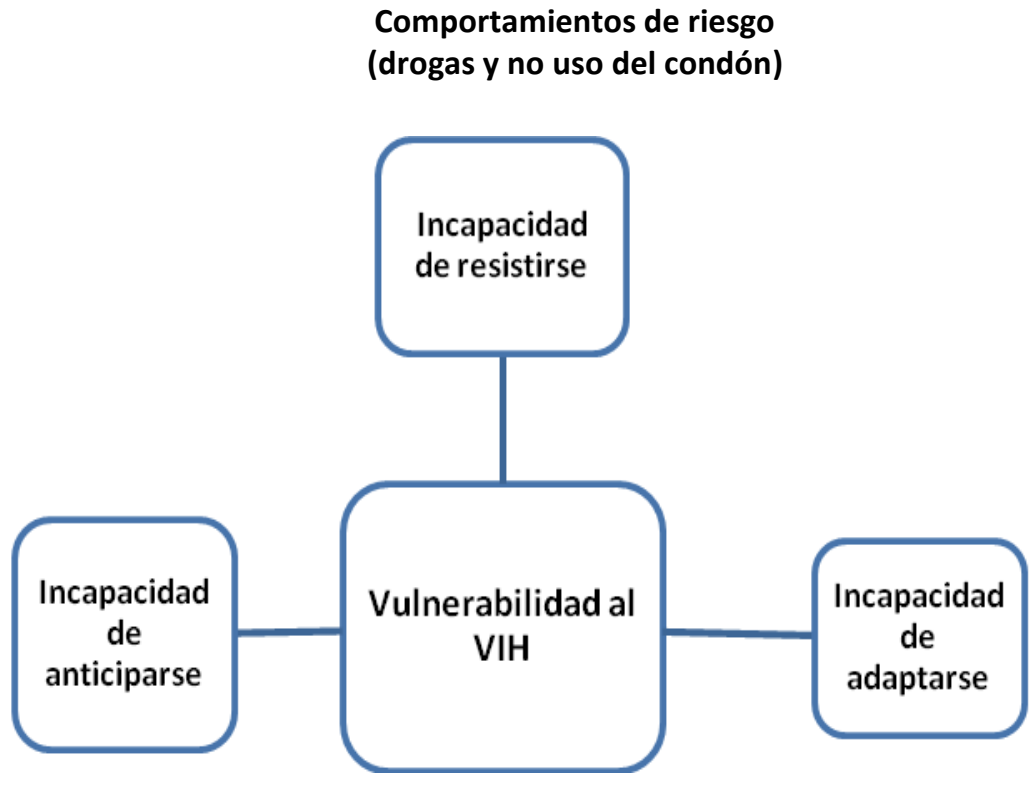

\begin{tabular}{|c|c|c|c|c|c|c|}
\hline & $\mathrm{X}^{2}$ & Df & $\mathrm{X}^{2} / \mathrm{df}$ & Valor de $\mathrm{p}$ & CFI & RMSEA \\
\hline Modelo 1 & 53,645 & 34 & 1,58 & 0,017 & 0,969 & 0,04 \\
\hline
\end{tabular}

El modelo en AFC logró ser significativo, y cumplir los requerimientos específicos de las mediciones, el modelo fue especificado utilizando como variables endógenas las variables observadas, y como variables exógenas los errores de los ítems y las variables latentes los tres factores propuestos (anticiparse, resistirse y recuperarse).

El valor CFI fue mayor de 0,90 , indicando un buen ajuste del modelo propuesto (cuanto mayor sea el valor, mejor ajuste); así mismo el valor RMSEA fue menor de $\leq 0,05$ (cuanto menor sea el valor, mejor ajuste). En este sentido, el modelo propuesto, reporta un ajuste adecuado presentando niveles admisibles de ajuste a los datos; igualmente todos los parámetros fueron significativos.

En este orden, para la presente investigación, la vulnerabilidad al VIH se define como la reducción de la capacidad de anticiparse (falta de conocimientos requeridos para protegerse y creencias erróneas), resistirse (prácticas de riesgo sexual y consumo de drogas) y recuperarse (falta de apoyo social, rechazo y estigmatización que desfavorece a ciertas poblaciones) limitando su capacidad para acceder a servicios de prevención, atención, y apoyo al VIH (Figura 2). 
Figura 2. Modelo de Vulnerabilidad al VIH

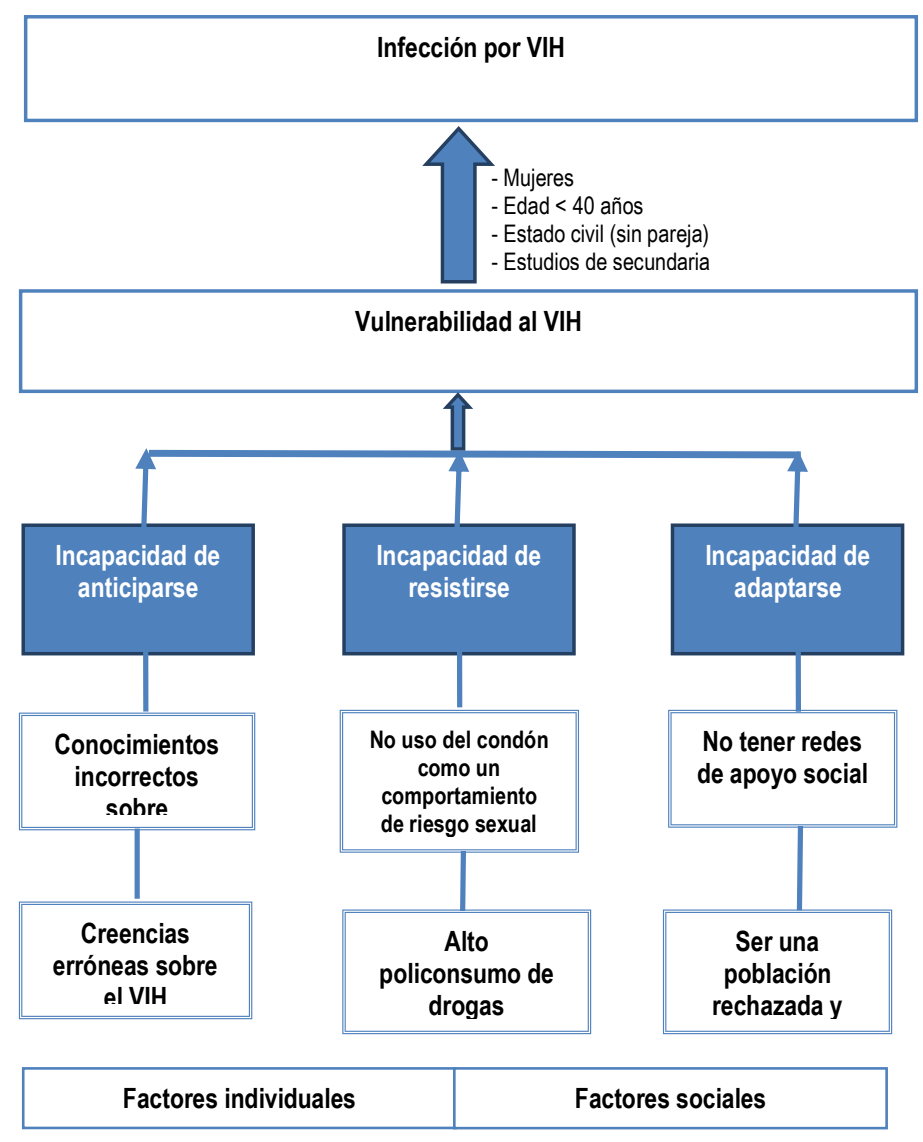

\section{Puntaje del Índice de Vulnerabilidad al VIH}

Inicialmente se consideraron tres categorías de análisis de vulnerabilidad al VIH (bajo, medio y alto) pero al tener una proporción tan baja en la primera categoría (bajo) se procedió a unir la categoría de vulnerabilidad baja-media $(60,1 \%)$ y compararlo con la vulnerabilidad alta $(39,9 \%)$ como categoría de referencia.

\section{Asociación entre VIH y Vulnerabilidad al VIH}

Se realizó un modelo multivariado de regresión logística con el fin de ajustar el riesgo de tener la infección por VIH con la variable creada de Vulnerabilidad al VIH. Se encontró que la población con alta vulnerabilidad al VIH tenía cinco veces el riesgo de tener infección por $\mathrm{VIH}$ (RPaj=4,51 [1,28-15,79]), esta asociación se ajustó por variables sociodemográficas de edad, sexo, estado civil y nivel de escolaridad (tabla 2). 
Tabla 2. Infección por VIH y Vulnerabilidad al VIH (Modelo ajustado por variables sociodemográficas)

\begin{tabular}{lccccc}
\hline \multicolumn{1}{c}{ Variable } & $\begin{array}{c}\mathbf{R P} \\
\text { crudo }\end{array}$ & IC 95\% & $\begin{array}{c}\mathbf{R P} \\
\text { ajustado }\end{array}$ & IC 95\% & Valor de $\mathbf{p}$ \\
\hline $\begin{array}{l}\text { Vulnerabilidad } \\
\quad \text { Alta }\end{array}$ & 3,2 & $1,05-9,78$ & 4,51 & $1,28-15,79$ & 0,01 \\
$\quad$ Baja-Media & 1 & - & 1 & - & \\
$\begin{array}{l}\text { Sexo } \\
\text { Femenino }\end{array}$ & 2,98 & $0,98-8,95$ & 2,24 & $0,59-8,48$ & 0,19 \\
$\quad$ Masculino & 1 & - & 1 & - & \\
Edad & & & & & \\
$\leq 40$ años & 2,02 & $0,66-6,17$ & 1,26 & $0,36-4,30$ & 0,71 \\
$>40$ años & 1 & - & 1 & - & \\
Estado civil & & & & & \\
$\quad$ Soltero & 5,13 & $1,55-16,99$ & 6,73 & $1,54-29,35$ & 0,01 \\
$\quad$ Otro & 1 & - & 1 & - & \\
Nivel de estudios & & & & & \\
$\quad \begin{array}{l}\text { Secundaria } \\
\quad \text { Primaria }\end{array}$ & 2,79 & $0,96-8,13$ & 3,99 & $1,16-13,66$ & 0,02 \\
\hline
\end{tabular}

\section{DISCUSIÓN}

Se ha reconocido que la infección por VIH tiende a ocurrir con las vulnerabilidades sociales ${ }^{(29)}$, pero pocos estudios han tratado de medir y explicar las formas en que los patrones de vulnerabilidad contribuyen al riesgo de esta infección. El presente artículo, propone un índice de vulnerabilidad que incorpora componentes descritos por la literatura que son: los conocimientos adecuados, las creencias erróneas y las actitudes sobre el $\mathrm{VIH}$; las redes de apoyo, el rechazo y discriminación, la frecuencia de consumo de drogas y el uso del condón, que permiten orientar acciones en contextos difíciles, como en el que se encuentran la población que habita de manera permanente en la calle.

En Colombia, la población habitante de calle no se encuentra priorizada como una población en riesgo de transmisión del VIH. Sin embargo, esta investigación identificó una prevalencia de la infección del $8,1 \%{ }^{(17)}$ que por las características de la epidemia de VIH/Sida en Colombia de naturaleza concentrada ${ }^{(30)}$ requieren la identificación de comportamientos de riesgo que permita conocer el aporte que podrían estar haciendo estos grupos al perfil actual y futuro de la epidemia de $\mathrm{VIH}^{(31)}$.

En un estudio realizado en México se exploró el modelo de Rogers ${ }^{(32)}$, para cuantificar la vulnerabilidad, se compararon los recursos personales y el apoyo ambiental, y tenía como base el grado de vulnerabilidad. Este enfoque identificó a la población analizada como muy sensible. La comparación entre el enfoque de riesgo y el propuesto mostró diferencias importantes, pero no permitió inferir cuál podía ser mejor. Con respecto a la investigación realizada en Medellín, en el modelo se incluyeron variables de apoyo social, y conocimientos adecuados para prevenir la infección por VIH. 
En Chile, se propuso una escala de vulnerabilidad para ser aplicada en trabajadores de la salud, constituida por cuatro dimensiones (conocimiento de la vulnerabilidad intrínseco, conocimiento de la vulnerabilidad extrínseca, experiencia de la vulnerabilidad y percepción de situaciones de vulnerabilidad en los pacientes) ${ }^{(33)}$. En el modelo propuesto para la población habitante de calle, se utilizaron los conocimientos de prevención del VIH como uno de los componentes, para determinar si estaba relacionado con el tener o no conductas riesgosas, encontrando diferencias importantes especialmente en las creencias inadecuadas sobre la transmisión del $\mathrm{VIH}$, los cuales pueden aumentar el rechazo por desconocimiento en las formas de transmisión hacia las personas con $\mathrm{VIH}$.

Utilizando el modelo de conducta, en un estudio sugieren que la vulnerabilidad social puede ser disminuida si las personas sin hogar logran encontrar una vivienda permanente, aliviar su enfermedad mental, y abstenerse del abuso de sustancias ${ }^{(20)}$; estas dos últimas categorías fueron utilizadas dentro del modelo propuesto de vulnerabilidad social al $\mathrm{VIH}$; las cuales tienen alta relación con la presencia de la infección.

El modelo de poblaciones vulnerables ampliado, incorpora tradicionales factores descritos en el modelo de Andersen (34,35) y también incluyen vulnerabilidades específicas comunes entre las personas sin hogar, tales como el abuso de sustancias, enfermedad mental, y las barreras para la atención de salud; estos tres componentes son similares a los observados en el análisis exploratorio realizado en el que los tres factores determinantes fueron el abuso de sustancias, la percepción de enfermedad, incluyendo la enfermedad mental y como otro factor el apoyo social y familiar descrito por otros autores en el marco de la vulnerabilidad al $\mathrm{VIH}{ }^{(11,36,37)}$.

La vulnerabilidad de una población con el VIH/Sida tiene sus raíces en los procesos sociales y las causas subyacentes que pueden realmente estar muy relacionadas con el resultado final en sí, es decir, la transmisión del VIH. En un modelo propuesto desde la teoría de los desastres, la vulnerabilidad al riesgo está determinada por tres factores:1) la capacidad de recuperación de una población, o la capacidad de las personas para resistir y recuperarse; 2) la salud de la población y disponibilidad de asistencia de servicios de salud y 3) la preparación de la población, este nivel de preparación está conformado por los valores y creencias sociales, que determinan lo que se considera un riesgo y, a su vez que se toman medidas para la protección; este modelo además reconoce que las poblaciones con mayor prevalencia de $\mathrm{VIH}$ son aquellas en las cuales persiste la desigualdad social y donde los desvalidos son victimizados ${ }^{(38)}$. Estos tres aspectos fueron analizados en el modelo propuesto para la población habitante de calle de Medellín, encontrando una consistencia interna alta que indica alta relación entre las variables.

Autores como Higgins et al. ${ }^{\left({ }^{39)}\right.}$ señalan que el uso del término "vulnerabilidad" marca la transición de un acercamiento individual de las prácticas de riesgo asociadas al $\mathrm{VIH}$, hacia un énfasis en los factores más allá del control del individuo; de manera similar, la presente investigación identificó un índice que recoge las prácticas y comportamiento de riesgo, y los factores sociales como la falta de apoyo social y el rechazo y la discriminación que limitan la capacidad de tomar decisiones.

Según ONUSIDA, los factores subyacentes a la vulnerabilidad pueden reducir la capacidad de los individuos y las comunidades para evitar el riesgo del VIH y pueden estar fuera del control de los individuos. Entre estos factores se incluyen, entre otros, 
la falta de conocimientos y las destrezas requeridas para protegerse a sí mismo y a los demás; la accesibilidad, la calidad y la cobertura de los servicios; y factores sociales como la violación de los derechos humanos o ciertas normas sociales y culturales ${ }^{(40)}$. Esta fue la definición con la cual se inició el análisis de la vulnerabilidad al VIH en la presente investigación y la que motivo la búsqueda sistemática de evidencia sobre el tema ${ }^{(14)}$; y fue partir de ella que se generó un concepto de vulnerabilidad al VIH que pretende ser aplicado a otras poblaciones.

Ayres et al., define el concepto de vulnerabilidad como "los diferentes grados y naturalezas de la susceptibilidad de los individuos y las colectividades a la infección o a enfermarse, que dependen de su situación frente al conjunto integrado de aspectos sociales e individuales que los ubican en relación con el problema y con los recursos para enfrentarlo"(41,42). Este autor especifica que los tres componentes de la vulnerabilidad al $\mathrm{VIH}$ son los factores individuales, sociales y programáticos, aunque esta última categoría es poco analizada en estudios que aplican este modelo teórico $^{(18,43,44)}$. Es decir, que aunque se reconoce que los factores programáticos pueden influir en la vulnerabilidad al VIH, su medición es compleja y para el caso de la presente investigación al tener una población altamente concentrada en algunos sectores y una comuna, no fue posible identificar variables contextuales relacionadas con el índice propuesto.

La explicación de la vulnerabilidad al VIH se ha orientado más hacia la comprensión de la compleja red de factores sociales que interactúan constantemente con las conductas de riesgo de VIH. Un tema clave en la literatura del VIH es la relación entre la desventaja social y vulnerabilidad SIDA ${ }^{(45)}$. El cual debe seguir siendo trabajado y considerado para orientar las acciones de salud pública.

Este estudio reporta las siguientes limitaciones que le generan restricción a los resultados expuestos pero no los invalidan, tales como: los datos provienen de una encuesta que recolectó auto-reportes de prácticas sexuales y de consumo de drogas, pudiendo tener imprecisiones basadas en la honestidad de la respuesta; no es posible realizar asociaciones de causa efecto entre la infección por el VIH y la vulnerabilidad al VIH debido al tipo de diseño, en los estudios transversales los datos del evento y la exposición son medidos en el mismo momento; y la metodología de tiempo y lugar es un muestreo no probabilístico, considerado un tipo de selección que debe cumplir una serie de supuestos para ser una muestra representativa ${ }^{(46)}$.

\section{REFERENCIAS}

1. Organización Mundial de la salud. Situación de la epidemia mundial de VIH. 2010 [Internet]. 2010. Recuperado a partir de: http://www.unaids.org/

2. Ministerio de Salud y Proteccion Social. Informe mundial de avances en la lucha contra el Sida. Colombia 2012 [Internet]. 2012. Recuperado a partir de: http://www.unaids.org/en/dataanalysis/knowyourresponse/countryprogressreports/201 2countries/

3. Ministerio de Protección Social. Vulnerabilidad a la infección por VIH. Ministerio de Proteccion Social; 2008.

4. Curran JW. The epidemiology and prevention of the acquired immunodeficiency syndrome. Ann Intern Med. noviembre de 1985;103(5):657-62.

5. Matuszak DL, Kotval I, Garg R, Patel J, Israel E, Glasser D. Prevalence of risk behaviors and HIV infection in Maryland STD clinics. Md Med J Baltim Md 1985.

diciembre de 1989;38(12):1019-26. 
6. Scala E, Luzi G, Aiuti F. [The correct use of the condom reduces the risk of HIV infection]. Immunol Clin. diciembre de 1989;8(4):201-13.

7. Kraft P, Rise J, Grønnesby JK. Prediction of sexual behaviour in a group of young Norwegian adults. NIPH Ann. diciembre de 1989;12(2):27-44.

8. Kalichman SC, Catz S, Ramachandran B. Barriers to HIV/AIDS treatment and treatment adherence among African-American adults with disadvantaged education. $J$ Natl Med Assoc. agosto de 1999;91(8):439-46.

9. Cargill VA, Stone VE. HIV/AIDS: a minority health issue. Med Clin North Am. julio de 2005;89(4):895-912.

10. Meyer DEE, Mello DF de, Valadão MM, Ayres JR de CM. «Você aprende. A gente ensina?»: interrogando relações entre educação e saúde desde a perspectiva da vulnerabilidade. Cad Saúde Pública. junio de 2006;22(6):1335-42.

11. Amirkhanian YA. Review of HIV vulnerability and condom use in central and eastern Europe. Sex Health. marzo de 2012;9(1):34-43.

12. Figueiredo R, Ayres JRCM. [Community based intervention and reduction of women's vulnerability to STD/AIDS in Brazil]. Rev Saúde Pública. agosto de 2002;36(4 Suppl):96-107.

13. Mawar N, Saha S, Pandit A, Mahajan U. The third phase of HIV pandemic: social consequences of HIV/AIDS stigma \& discrimination \& future needs. Indian J Med Res. diciembre de 2005;122(6):471-84.

14. Berbesi-Fernandez DYB, Cardona ÁS. Vulnerabilidad al VIH: Revisión sistemática. Investig ANDINA. 1 de abril de 2014;16(28):964-77.

15. Ayres JR de CM, Paiva V, França I Jr, Gravato N, Lacerda R, Della Negra M, et al. Vulnerability, human rights, and comprehensive health care needs of young people living with HIV/AIDS. Am J Public Health. junio de 2006;96(6):1001-6.

16. Katzman R. Notas sobre la medición de la vulnerabilidad social. Documentos de Trabajo de IPES [Internet]. [citado 5 de mayo de 2013]. Recuperado a partir de: http://www.eclac.cl/deype/mecovi/docs/taller5/24.pdf

17. Berbesi D, Segura-Cardona A, Caicedo B, Cardona-Arango D. Prevalencia y factores asociados al VIH en habitante de calle de la ciudad de Medellín Colombia. Fac Nac Salud Pública. 15 de mayo de 2015;33(2):200-5.

18. Urzúa R, Zuñiga P. Vulnerabilidad al VIH en mujeres en riesgo social. Rev Saúde Pública [Internet]. 2008;1. Recuperado a partir de:

http://www.scielosp.org/pdf/rsp/2008nahead/6903.pdf

19. Ministerio de la Proteccion Social. Republica de Colombia. Vulnerabilidad a la infección por VIH en personas en situación de calle en la ciudades de Bucaramanga y Barranquilla. 2011.

20. Oliveira JF de, Paiva MS. Vulnerabilidade de mulheres usuárias de drogas ao HIV/AIDS em uma perspectiva de gênero. Esc Anna Nery. diciembre de 2007;11(4):625-31.

21. Alcaldia de Medellin. - Ciudadanos - Bienestar Social - Información Secretarías - Secretaría de Inclusión Social y Familia [Internet]. 2014 [citado 19 de julio de 2013]. Recuperado a partir de:

http://www.medellin.gov.co/irj/portal/ciudadanos?NavigationTarget=navurl://c5d04e891 6d98b82a2c697e214ce9c61

22. Berbesi D, Agudelo A, Segura C, Montoya L. VIH en habitantes de calle de Medellín. Rev Fac Nac Salud Publica. 2012;30:310-5.

23. Grisales R H, M A, P M. Methodology for the construction and validation of an index of life conditions for young adolescents. Rev Fac Nac Salud Pública. julio de 2008;26(2):178-95. 
24. Grisales Romero H, Márquez F, Rojas JC. Construction of a development index for young people aged 14 - 26 in Medellín, Colombia. Rev Panam Salud Pública. abril de 2014;35(4):242-7.

25. Reducción de Datos: Análisis de Componentes Principales y Factorial.

[Internet]. [citado 16 de noviembre de 2014]. Recuperado a partir de:

http://web.ua.es/es/lpa/docencia/practicas-analisis-exploratorio-de-datos-conspss/practica-5-analisis-multivariante-con-spss-reduccion-de-datos-analisis-decomponentes-principales-y-factorial.html

26. Berbesi-Fernandez D, Montoya-Vélez, Segura-Cardona. Uso del condón y conocimientos sobre prevención del VIH entre los inyectadores de drogas de dos ciudades colombianas. Adicciones. 2013;25(4):321-6.

27. Segura-Cardona Á, Berbesí-Fernández D, Cardona-Arango D, Ordóñez-Molina J. Construcción preliminar de un cuestionario sobre conocimientos en VIH/SIDA en excombatientes de Colombia. Rev Peru Med Exp Salud Publica. julio de $2011 ; 28(3): 503-7$.

28. Artiles López DM, Sangabriel AS. Construyendo la vulnerabilidad:: Un riesgo para todos. Arquit Urban. agosto de 2012;33(2):68-78.

29. USAID. Conocimiento de VIH/SIDA, Otras ETS y Formas de Prevención [Internet]. 2010. Recuperado a partir de: http://www.measuredhs.com/

30. Organizacion Mundial de la Salud, ONUSIDA. Guías sobre la vigilancia del VIH de segunda generacíon [Internet]. 2000. Recuperado a partir de:

http://cedoc.cies.edu.ni/general/2nd_Generation\%20\%28D\%29/Surveillance\%20Guid elines/2nd\%20Generation\%20Surveillance/2nd\%20gen\%20Spa.pdf

31. Ministerio de la Proteccion Social. Republica de Colombia. Plan Nacional de respuesta ante el VIH y el SIDA. Colombia 2008 - 2011. 2008.

32. Rogers AC. Vulnerability, health and health care. J Adv Nurs. 1 de julio de $1997 ; 26(1): 65-72$.

33. Cabieses B, Ferrer L, Tunstall H, Norr K. The relationship between knowledge of HIV, self-perceived vulnerability and sexual risk behavior among community clinic workers in Chile. Rev Salud Publica. 12(5):777-89.

34. Andersen RM. A behavioral model of families' use of health services. 1968;(25):xi + $111 \mathrm{pp}$.

35. Andersen RM. National Health Surveys and the Behavioral Model of Health Services Use: Med Care. julio de 2008;46(7):647-53.

36. Arcos E, Muñoz LA, Sánchez X, Vollrath A, Latorre C, Bonatti C, et al. Vulnerabilidad social en mujeres embarazadas de una comuna de la Región Metropolitana. Rev Médica Chile. junio de 2011;139(6):739-47.

37. DeFur PL, Evans GW, Cohen Hubal EA, Kyle AD, Morello-Frosch RA, Williams DR. Vulnerability as a function of individual and group resources in cumulative risk assessment. Environ Health Perspect. mayo de 2007;115(5):817-24.

38. Tsasis Peter, Nirupama N. Vulnerability and risk perception in the management of HIV/AIDS: Public priorities in a global pandemic. Risk Manag Heal Policy. 2008;1:714.

39. Higgins JA, Hoffman S, Dworkin SL. Rethinking gender, heterosexual men, and women's vulnerability to HIVIAIDS. Am J Public Health. marzo de 2010;100(3):435-45. 40. ONUSIDA. Orientaciones terminológicas de ONUSIDA [Internet]. 2012.

Recuperado a partir de:

http://www.unaids.org/en/media/unaids/contentassets/documents/unaidspublication/20 11/JC2118_terminology-guidelines_es.pdf

41. Ayres JR de CM, França Júnior I, Calazans GJ, Filho S, César H. O conceito de vulnerabilidade e as práticas de saúde: novas perspectivas e desafios [Internet].

FIOCRUZ; 2003 [citado 1 de octubre de 2014]. Recuperado a partir de: 
http://bases.bireme.br/cgi-

bin/wxislind.exe/iah/online/?IsisScript=iah/iah.xis\&src=google\&base=LILACS\&lang=p\& nextAction=Ink\&exprSearch $=350313$ \&indexSearch=ID

42. Ayres JR de CM, Júnior F, França I, Calazans GJ, Filho S, César H.

Vulnerabilidade e prevençäo em tempos de Aids [Internet]. Editora 34; 1999 [citado 1 de octubre de 2014]. Recuperado a partir de: http://bases.bireme.br/cgi-

bin/wxislind. exe/iah/online/?IsisScript=iah/iah. $x$ is\&src=google\&base=LILACS\&lang=p\& nextAction=Ink\&exprSearch=242122\&indexSearch=ID

43. Sousa PKR de, Miranda KCL, Franco AC. Vulnerabilidade: análise do conceito na prática clínica do enfermeiro em ambulatório de HIV/AIDS. Rev Bras Enferm. abril de $2011 ; 64(2): 381-4$.

44. Silva TMR da, Alvarenga MRM, Oliveira MA de C. Evaluation of the vulnerability of families assisted in Primary Care in Brazil. Rev Lat Am Enfermagem. octubre de 2012;20(5):935-43.

45. German D, Latkin CA. Social stability and HIV risk behavior: evaluating the role of accumulated vulnerability. AIDS Behav. enero de 2012;16(1):168-78.

46. Sabin KM, Johnston LG. Epidemiological challenges to the assessment of HIV burdens among key populations: respondent-driven sampling, time-location sampling and demographic and health surveys. Curr Opin HIV AIDS. marzo de 2014;9(2):101-6.

Recibido: 26 de febrero 2016;

Aceptado: 6 de mayo 2016 